Cite this: Soft Matter, 2014, 10, 446

\title{
Tuning biaxiality of nematic phases of board-like colloids by an external magnetic field
}

\begin{abstract}
Anke B. G. M. Leferink op Reinink, ${ }^{\text {a }}$ Simone Belli, ${ }^{\text {b }}$ René van Roij, ${ }^{\mathrm{b}}$ Marjolein Dijkstra, ${ }^{c}$ Andrei V. Petukhov and Gert Jan Vroege ${ }^{a}$

We study the influence of a magnetic field on the biaxial nematic phase of board-like goethite colloids both experimentally and theoretically. Using synchrotron small angle X-ray scattering techniques we find that applying a magnetic field along the main director of the biaxial nematic phase leads to a clear decrease in biaxiality with increasing magnetic field strength. Above a certain magnetic field strength the biaxiality is completely suppressed and the biaxial nematic phase transforms into an ordinary prolate uniaxial nematic phase. In order to interpret the physical mechanism behind this phenomenon, we develop a mean-field theory for the liquid crystal phase behaviour of the suspension. Within this theory the magnetic properties of the particles are modelled by taking into account the effect of both the permanent and the induced magnetic dipoles. The resulting phase diagrams support our experimental findings of the field-induced biaxial nematic to prolate uniaxial nematic transition. They additionally predict that for more plate-like particles, which initially would only display oblate nematic ordering of the shortest axis, the rare biaxial phase can be induced by applying a magnetic field with a carefully chosen field strength, a parameter which can be easily tuned.

Received 22nd August 2013

Accepted 1st November 2013

DOI: $10.1039 / \mathrm{c} 3 \mathrm{sm} 52242 \mathrm{c}$

www.rsc.org/softmatter
\end{abstract}

He showed that at sufficiently high volume fraction the loss in orientational entropy is compensated by the gain in excluded volume entropy.

There exist different nematic phases. In the usual (uniaxial) nematic phase the particles' main axes orient along a common direction. This is either the long particle axis in rod-like systems, the resulting nematic being the prolate uniaxial nematic $\left(\mathrm{N}_{+}\right)$phase, or the short particle axis in plate-like systems, alignment of which results in the oblate uniaxial nematic phase $\left(\mathrm{N}_{-}\right)$. Additionally, there is the biaxial nematic $\left(\mathrm{N}_{\mathrm{B}}\right)$ phase, in which particles orient along both axes. The appearance of this $\mathrm{N}_{B}$ phase in the phase diagram was theoretically predicted by Freiser ${ }^{5}$ in 1970 and immediately attracted significant attention ${ }^{6,7}$ since the improved alignment makes biaxial nematic phases promising candidates for LCDs. Unfortunately, the biaxial nematic phase is rare. There are few experimental examples, most of which are still under debate. ${ }^{\mathbf{8 - 1 0}}$

Board-like colloidal particles of the mineral goethite $(\alpha-\mathrm{FeOOH})$ constitute one of the few known experimental realizations ${ }^{11}$ of the $\mathrm{N}_{\mathrm{B}}$ phase. Above a volume fraction of typically 0.1 , the goethite particles - characterized by their length $L>$ width $W>$ thickness $T$ - not only spontaneously orient their $L$-axis but their $W$-and $T$-axes as well. Depending on the degree of polydispersity and volume fraction of the particles, this can either be a biaxial nematic phase, a biaxial smectic phase (in which the particles are stacked in layers), or both. ${ }^{11-13}$

Understanding the factors determining the formation of biaxial liquid crystal phases is important for possible
${ }^{a}$ Van't Hoff Laboratory for Physical and Colloid Chemistry, Debye Institute for Nanomaterials Science, Utrecht University, Padualaan 8, 3584CH Utrecht, The Netherlands. E-mail: A.B.G.M.LeferinkopReinink@uu.nl; G.J.Vroege@uu.nl; Fax: +31-30-(253)38 70; Tel: +31-30-(253)2391

${ }^{b}$ Institute for Theoretical Physics, Utrecht University, Leuvenlaan 4, 3584CE Utrecht, The Netherlands

${ }^{c}$ Soft Condensed Matter, Debye Institute for Nanomaterials Science, Utrecht University, Princetonplein 5, 3584CC Utrecht, The Netherlands 
applications. ${ }^{\mathbf{1 4}}$ Biaxial nematic stability can be interpreted as a balanced competition between rod-like and plate-like behaviour. Theoretically, biaxial phases are found for specific particle dimensions close to the condition $(L / W)=(W / T)$, hence for particles with a shape in between rod- and plate-like. ${ }^{15}$ However for the goethite system unexpectedly a direct $\mathrm{I}-\mathrm{N}_{\mathrm{B}}$ phase transition was also found for slightly elongated board-like particles with a shape parameter $(v \equiv(L / W)-(W / T))$ of $0.1{ }^{11}$ Theory explained $^{\mathbf{1 6}}$ that the stability of the goethite $\mathrm{N}_{\mathrm{B}}$ phase could be attributed to the system's high polydispersity ( $25 \%)$ which leads, similar to the effect of a depletant, ${ }^{17}$ to $\mathrm{N}_{+}-\mathrm{N}_{-}$ competition.

Colloidal goethite particles have very peculiar magnetic properties which make them highly susceptible to an external magnetic field. ${ }^{18}$ They possess a permanent magnetic moment along their long axis, but their magnetic easy axis is perpendicular to this axis. ${ }^{\mathbf{1 8}}$ Therefore, particles align parallel to a weak magnetic field. However, above a certain magnetic field strength, called the critical magnetic field strength $\left(B^{*}\right)$, the induced magnetic moment is larger than the permanent moment. As a result, the particles reorient to align their long axis in the direction perpendicular to the magnetic field. ${ }^{19-23}$ Since the particles easily align in a (weak) magnetic field, magnetic fields are often used to reorient goethite (biaxial nematic) liquid crystal phases..$^{23,24}$

In this paper we investigate the effect of a magnetic field on the stability of the goethite biaxial nematic phase both experimentally, using small angle X-ray scattering (SAXS), and theoretically, by means of a mean-field theory. In Section 2 we describe the experimental goethite system, sample preparation and the SAXS set-up. In Section 3 we describe the theoretical framework: a mean-field theory where the magnetic properties of the particles are modelled taking into account the effect of the permanent and induced magnetic moments. Sections 4 and 5 then describe the experimental and theoretical results, respectively. Finally, in Section 6 we draw our conclusions.

\section{Experimental}

\subsection{Sample preparation}

Goethite particles were obtained by hydrolysis of iron nitrate at high $\mathrm{pH}$, according to the procedure described by Lemaire et $a l .{ }^{19}$ The particles were washed with Millipore (mp) water and charged in a $3 \mathrm{M} \mathrm{HNO}_{3}$ (Merck, 65\%, p.a.) solution. After three repetitive steps of washing and redispersion in $\mathrm{mp}$ water the particles were dispersed in slightly acidified (with $\mathrm{HNO}_{3}$ ) $\mathrm{mp}$ water to obtain a stable dispersion at $\mathrm{pH}=3$. No additional salt was added, hence the salt concentration is solely determined by the $\mathrm{H}^{+}$and charge-compensating $\mathrm{NO}_{3}{ }^{-}$ions. The Debye length is then $10 \mathrm{~nm}$ at most.

A Technai 10 (FEI company) transmission electron microscope was used to determine particle size distributions. The lengths and widths of 600 particles were measured using iTEM imaging software. The thickness of the particles is harder to measure because the particles have a tendency to lie on their $L / W$ face. Therefore the thickness was measured of 150 particles. The average particle dimensions were found to be $L \times W \times$
$T=254 \times 83 \times 28 \mathrm{~nm}$ with a polydispersity of $25 \%$ in all dimensions. The shape parameter $v \equiv(L / W-W / T) \approx 0.1$. Since $T$ was measured independently from $W$ an estimate of the variation in $v$ cannot be given. ${ }^{25}$

A flat glass capillary with internal dimensions of $0.2 \times 4.0 \times$ $100 \mathrm{~mm}^{3}$ (Vitrocom W3520-100) was filled with the goethite dispersion. The initial goethite volume fraction $\left(\phi_{\mathrm{g}}\right)$ was $12.5 \%$. The capillary was flame sealed and stored in an upright position to assure the formation of a density gradient by the gravitational field of the earth.

\subsection{Small angle X-ray scattering measurements}

SAXS patterns were collected using the microradian diffraction set up $^{26}$ at the BM26 DUBBLE beamline ${ }^{27}$ of the European Synchrotron Radiation Facility (ESRF, Grenoble, France). A photon energy of $12 \mathrm{keV}$ was used, with a sample-detector distance of $7 \mathrm{~m}$. Patterns were collected using a two-dimensional X-ray CCD-based detector with a pixel size of $22 \mu \mathrm{m}$ (Photonic Science). Two homemade variable permanent magnets were used to apply magnetic fields parallel or perpendicular to the X-ray beam. By changing the distance between the poles, the field strength could be varied from 3 to $\sim 700 \mathrm{mT}$ for both magnets. Patterns were collected in the middle of the capillary every mm along the vertical $(y)$ direction using a motorized translational stage. Relative goethite volume fractions were determined from the X-ray adsorption measured at different heights in the sample.

\section{Theoretical framework}

We developed a mean-field density functional theory to predict the phase behaviour of hard board-like particles under the effect of a magnetic field. By calculating the excluded volume between two uniaxial rods as a function of their orientation, Onsager was able to predict the stability of a nematic liquid crystal phase. ${ }^{4}$ Unfortunately, the corresponding expression for the excluded volume of a pair of board-like particles is not available. Hence, in the present analysis we circumvent the problem by adopting a discrete-orientation model. ${ }^{28}$

In ref. 29 it is shown that the isotropic-nematic transition of uniaxial rods remains first-order if the number of allowed orientations is gradually decreased from infinity (Onsager's continuum) ${ }^{4}$ to three (the Zwanzig model of rods). ${ }^{28}$ Although a similar study for board-like particles has not been performed yet, the results of ref. 29 suggest that the nature of the isotropicnematic transition is robust with discretising the orientational degrees of freedom. Note, however, that the location of the binodal, the magnitude of the phase gap, and the nematic ordering of the coexisting nematic state in ref. 29 all do depend on the degree of discretization. Therefore, one should expect the predictions of the discrete-orientation model for the present system to be mainly qualitative.

Within this model the total number of orientations is reduced to the $i=1, \ldots, 6$ independent ways a board-like particle can align its main axes along those of a fixed Cartesian reference frame. Moreover, we assume each particle to carry a 
permanent magnetic dipole directed along the longest axis. We indicate with $\alpha=+$, - when the magnetic dipole is oriented parallel and antiparallel to the Cartesian axis, respectively. As a result, the orientation of a single board-like particle can be

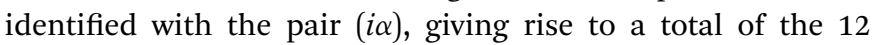
independent orientations depicted in Fig. 1.

\subsection{Density functional theory}

We consider a system of $N$ identical board-like particles with dimensions $L \times W \times T$ in a volume $V$ at room temperature $T$ under the effect of the homogeneous external energy field $u_{i \alpha}$. Let us indicate with $\rho_{i \alpha}$ the number density of particles with orientation $(i \alpha)$ in a homogeneous phase, satisfying the normalization

$$
\sum_{i, \alpha} \rho_{i \alpha}=\frac{\phi}{v}
$$

where $\phi=N v / V$ is the particle volume fraction and $v=L \times W \times$ $T$ is the single-particle volume. The free-energy functional per unit volume is defined as $^{30}$

$$
\beta f\left[\rho_{i \alpha}\right]=\sum_{i, \alpha} \rho_{i \alpha}\left[\log \left(\rho_{i \alpha} \Lambda^{3}\right)-1\right]+\beta f_{\mathrm{exc}}\left[\rho_{i \alpha}\right]+\sum_{i, \alpha} \rho_{i \alpha} \beta u_{i \alpha}
$$

where $\beta=\left(k_{\mathrm{B}} T\right)^{-1}, k_{\mathrm{B}}$ is the Boltzmann constant and $\Lambda$ is the thermal wavelength. The first term on the right-hand side of eqn (2) represents the ideal-gas contribution, whereas $f_{\text {exc }}$ is the excess free energy per unit volume due to particle-particle interactions. The excess free energy cannot be evaluated exactly and hence has to be approximated. In the present study we employ the second-virial approximation, according to which

$$
\beta f_{\text {exc }}\left[\rho_{i \alpha}\right]=\frac{G(\phi)}{2} \sum_{i, i^{\prime}} E_{i i^{\prime}}\left(\rho_{i+}+\rho_{i-}\right)\left(\rho_{i^{\prime}+}+\rho_{i^{\prime}-}\right),
$$
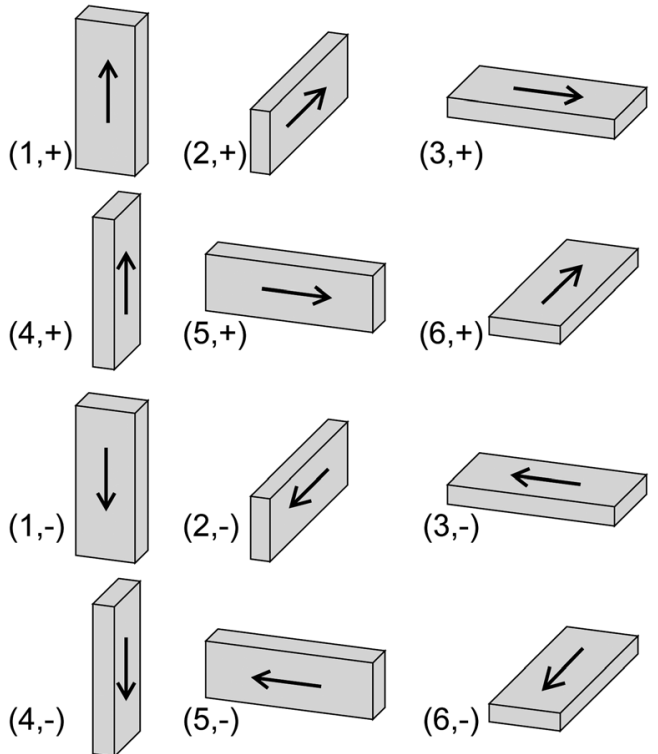

$(2,-)$

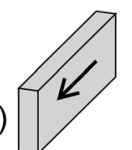

$(3,-)$
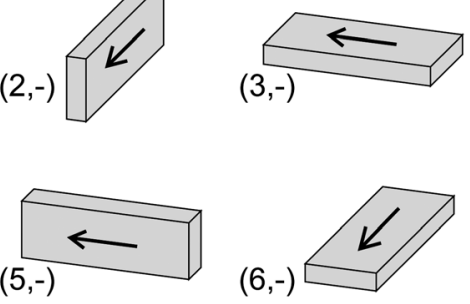

$(6,-)$

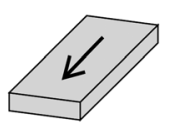

Fig. 1 Orientational degrees of freedom of a magnetic board-like particle in the restricted-orientation model. where $E_{i i^{\prime}}$ is the excluded volume of two particles with orientations $i$ and $i^{\prime}$, respectively, due to the interparticle repulsion. ${ }^{16,17}$ In the present model magnetic interactions between particles are disregarded due to the relatively negligible strength of their magnetic dipoles. The term $G(\phi)=$ $(1-3 / 4 \phi) /(1-\phi)^{2}$ in eqn (3) is a Parsons-Lee correction term, ${ }^{31-33}$ introduced in order to reduce the quantitative deviations expected in the second-virial approach. Finally, the last term on the right-hand side of eqn (2) represents the external energy contribution to the free energy of the system, which is discussed in the following section. Once an approximation for the excess free energy $f_{\text {exc }}$ is established, the equilibrium single-particle density is found as the minimum of eqn (2) under the constraint of eqn (1). In other words, the equilibrium single-particle density at the packing fraction $\phi$ is calculated by solving the Euler-Lagrange equation

$$
\rho_{i \alpha}=\frac{\phi}{v} \frac{\exp \left[-G(\phi) \sum_{i^{\prime}, \alpha^{\prime}} E_{i i^{\prime}} \rho_{i^{\prime} \alpha^{\prime}}-\beta u_{i \alpha}\right]}{\sum_{i^{\prime \prime}, \alpha^{\prime \prime}} \exp \left[-G(\phi) \sum_{i^{\prime}, \alpha^{\prime}} E_{i^{\prime \prime} i^{\prime}} \rho_{i^{\prime} \alpha^{\prime}}-\beta u_{i^{\prime \prime} \alpha^{\prime \prime}}\right]} .
$$

Due to its non-linear character eqn (4) cannot be solved analytically. However, standard algorithms such as the Picard method can be applied to its numerical solution.

From the knowledge of the equilibrium single-particle density $\rho_{i \alpha}$ one can investigate the broken symmetries of the phase point under consideration by calculating the nematic order parameters. In case the nematic director is the vertical axis in Fig. 1, the (prolate) uniaxial nematic order parameter can be expressed as

$$
\text { U.O.P. }=\frac{v}{\phi} \sum_{\alpha}\left\{\rho_{1 \alpha}+\rho_{4 \alpha}-\frac{1}{2}\left[\rho_{2 \alpha}+\rho_{3 \alpha}+\rho_{5 \alpha}+\rho_{6 \alpha}\right]\right\} .
$$

This order parameter takes positive values in the case of prolate nematic ordering, while negative values for oblate nematic. On the other hand, a zero value describes an isotropic phase. Additionally, in order to take into account the possibility of biaxial ordering, one has to introduce a biaxial order parameter, which we define as

$$
\text { B.O.P. }=\frac{v}{\phi}\left|\sum_{\alpha} \rho_{1 \alpha}-\rho_{4 \alpha}\right| .
$$

\subsection{Magnetic energy}

Let us consider the energy of a single goethite particle in a constant external magnetic field with strength $B$ directed along the $z$ axis. Let $m=1.7 \times 10^{-20} \mathrm{~A} \mathrm{~m}^{2}$ be the strength of the particle's permanent magnetic dipole. ${ }^{34}$ By referring to Fig. 1, one finds that in the absence of an induced magnetic dipole the magnetic energy in the present discrete-orientation model reads

$$
u_{i \alpha}=-m B\left(\delta_{i 1}+\delta_{i 4}\right)\left(\delta_{\alpha+}-\delta_{\alpha-}\right) \text {, }
$$


where $\delta$ is the Kronecker delta. On the other hand, most of the peculiar magnetic properties of goethite particles are due to the presence of an induced magnetic dipole. ${ }^{18,20,21}$ Such an induced dipole is known to develop in the plane perpendicular to the permanent dipole, that is, along the shortest and the intermediate particle axes. In order to account for such an induced magnetic dipole, eqn (7) has to be substituted by the following expression

$$
u_{i \alpha}=-m B\left(\delta_{i 1}+\delta_{i 4}\right)\left(\delta_{\alpha+}-\delta_{\alpha-}\right)-K B^{2}\left(\delta_{i 2}+\delta_{i 3}+\delta_{i 5}+\delta_{i 6}\right),
$$

where

$$
K=\frac{v|\Delta \chi|}{2 \mu_{0}} .
$$

In the previous equations $v=L \times W \times T$ is the single-particle volume, $\mu_{0}=4 \pi \times 10^{-7} \mathrm{~N} \mathrm{~A}^{-2}$ the magnetic permeability and $\Delta \chi=\chi_{\|}-\chi_{\perp}$ the susceptibility anisotropy with respect to the long axis of the particle. Experimental measurements for the susceptibility anisotropy of such particles exist and suggest a value varying between $-3 \times 10^{-4}$ (in ref. 19) and $-7 \times 10^{-4}$ (in ref. 34). In principle, one could plug these values into eqn (9) to obtain an estimate for the coefficient $K$. In practice, however, the large uncertainty of the particle volume $v$ due to the relatively high degree of polydispersity of the sample does not allow for a sufficiently accurate estimate of $K$. In order to overcome this limitation, we apply our model to predict the value of the constant $K$. We chose $K$ such that at room temperature in the dilute limit the critical magnetic field takes the experimental value of $250 \mathrm{mT}$. The corresponding value of $K$ was found to be $K=2.9 \times 10^{-20} \mathrm{~A}^{2} \mathrm{~m}^{4} \mathrm{~J}^{-1}$. This corresponds to a susceptibility anisotropy of $-1.2 \times 10^{-4}$ which is close to the values found in the literature.

\section{Experimental results}

The sample was allowed to sediment for three months before SAXS patterns were taken. The liquid crystal phases formed in the sample were first characterized in a very weak magnetic field of $3 \mathrm{mT}$ applied parallel to the X-ray beam. This is the lowest field possible with the magnet poles at maximum spacing. At low volume fractions, such a weak field might partially direct the main director along the field but does not change the liquidcrystal phase itself. SAXS patterns were taken every $0.5 \mathrm{~mm}$ along the $y$-direction (Fig. 2) of the capillary and revealed an isotropic phase on top of a $3 \mathrm{~mm}$ high nematic sediment. The left column in Fig. 2 shows the collected SAXS patterns taken in a magnetic field of $3 \mathrm{mT}$ at three different heights in the sediment: at the capillary bottom $(y=0 \mathrm{~mm})$ and at 1.5 and $2 \mathrm{~mm}$ above the capillary bottom. All three patterns show similar diffuse reflections characteristic of a (biaxial) nematic phase. The patterns are highly anisotropic; two orthogonal peaks can be observed at large scattering vectors. The radial profiles corresponding to the SAXS-pattern taken at $y=0 \mathrm{~mm}$ (black line in Fig. 3(b)) reveal $Q$-values of $0.045(140 \mathrm{~nm})$ and $0.086 \mathrm{~nm}^{-1}(73$ $\mathrm{nm}$ ) respectively. They originate from the correlations of the $W$ and $T$ dimensions of the particles, respectively. The ratio between the $Q$-values of the two peaks $Q_{T} / Q_{W}=1.9$ agrees reasonably well with the particle aspect ratio $W / T$ taking into account the Debye length $(\sim 10 \mathrm{~nm})$. Additionally, the sample had been sedimenting for only three months, which means that $\phi_{\mathrm{g}}$ is relatively low and thus the inter-particle spacing is large compared to the particle dimensions. For the pattern taken at $y=2 \mathrm{~mm}$ (Fig. 3(d)) reflections are found at slightly smaller $Q$-values. Due to sedimentation the goethite volume fraction decreases with the height in the sample $\left(\phi_{g, y=0}=1.27 \times \phi_{g, y=2}\right)$, hence the particle spacing is slightly larger at $y=2 \mathrm{~mm}$. Azimuthal profiles of the scattering intensity along circles at the $Q$-values corresponding to the correlation peaks are shown in Fig. 3(a) and (c). The different correlation peaks clearly appear along orthogonal directions of each other. From the plots and the patterns it is clear that the particles are orientationally ordered in three dimensions hence the patterns taken at $3 \mathrm{mT}$ originate from the biaxial nematic liquid crystal phase $\left(\mathrm{N}_{\mathrm{B}}\right)$ (Fig. 2). ${ }^{11}$

To study the influence of an external magnetic field on the $\mathrm{N}_{\mathrm{B}}$ phase SAXS-patterns were taken while increasing the magnetic field strength stepwise. The measurements were taken at the same heights in the capillary as before. The magnetic field strength ranges from 40 to $120 \mathrm{mT}$. This is far below the critical magnetic field strength, hence the particles align their long axis along the field. The resulting SAXS patterns are shown in Fig. 2. For the patterns taken at $y=0 \mathrm{~mm}$, there is no significant change due to the increase of the magnetic field strength. Compared to the pattern taken at $3 \mathrm{mT}$, a subtle increase in intensity can be observed, as well as a slight change in the directions in which the reflections can be found. This is due to the particles aligning in the field. Similar conclusions can be drawn from the radial profiles (Fig. 3(a) and (b)). Fig. 3(b) shows the subtle increase in intensity for the patterns taken at $B$ $>3 \mathrm{mT}$. However, the positions of the peaks are unchanged. The azimuthal profiles (Fig. 3(a)) confirm this, as well as the slight change in orientation of the nematic reflections. The observed reflections however still clearly indicate the biaxial ordering of the nematic phase for all magnetic field strengths. Whereas the magnetic field effects on the $\mathrm{N}_{B}$ phase at $y=0 \mathrm{~mm}$ are small, the effect on the $\mathrm{N}_{\mathrm{B}}$ phases is significant at $y=1.5$ and $2 \mathrm{~mm}$, where the volume fraction $\phi_{\mathrm{g}}$ is lower. For the patterns taken at $y=1.5 \mathrm{~mm}$, the anisotropy of the pattern decreases when the magnetic field strength increases (Fig. 2). This indicates that the actual biaxial order decreases upon increasing the magnetic field. The sharp character of this transition is manifested upon going from 40 to $80 \mathrm{mT}$.

The decrease in biaxial ordering is even more pronounced for the patterns taken at $y=2 \mathrm{~mm}$; at 80 and $120 \mathrm{mT}$ the patterns appear to be isotropic. This indicates that the particles lost their biaxial ordering and formed a prolate uniaxial nematic phase $\left(\mathrm{N}_{+}\right)$instead (schematic drawing Fig. 2). The magnetic field-induced $\mathrm{N}_{\mathrm{B}}$ to $\mathrm{N}_{+}$transition observed at $y=2$ $\mathrm{mm}$ is confirmed by the radial and azimuthal profiles shown in Fig. $3(\mathrm{c})$ and $(\mathrm{d})$. The $I(Q)$ plots originating from the pattern taken at $120 \mathrm{mT}$ given in Fig. 3(d) show that the combined reflections of both the $W$ and $T$ correlations of the particles are present in both orthogonal directions. The radial profiles are 


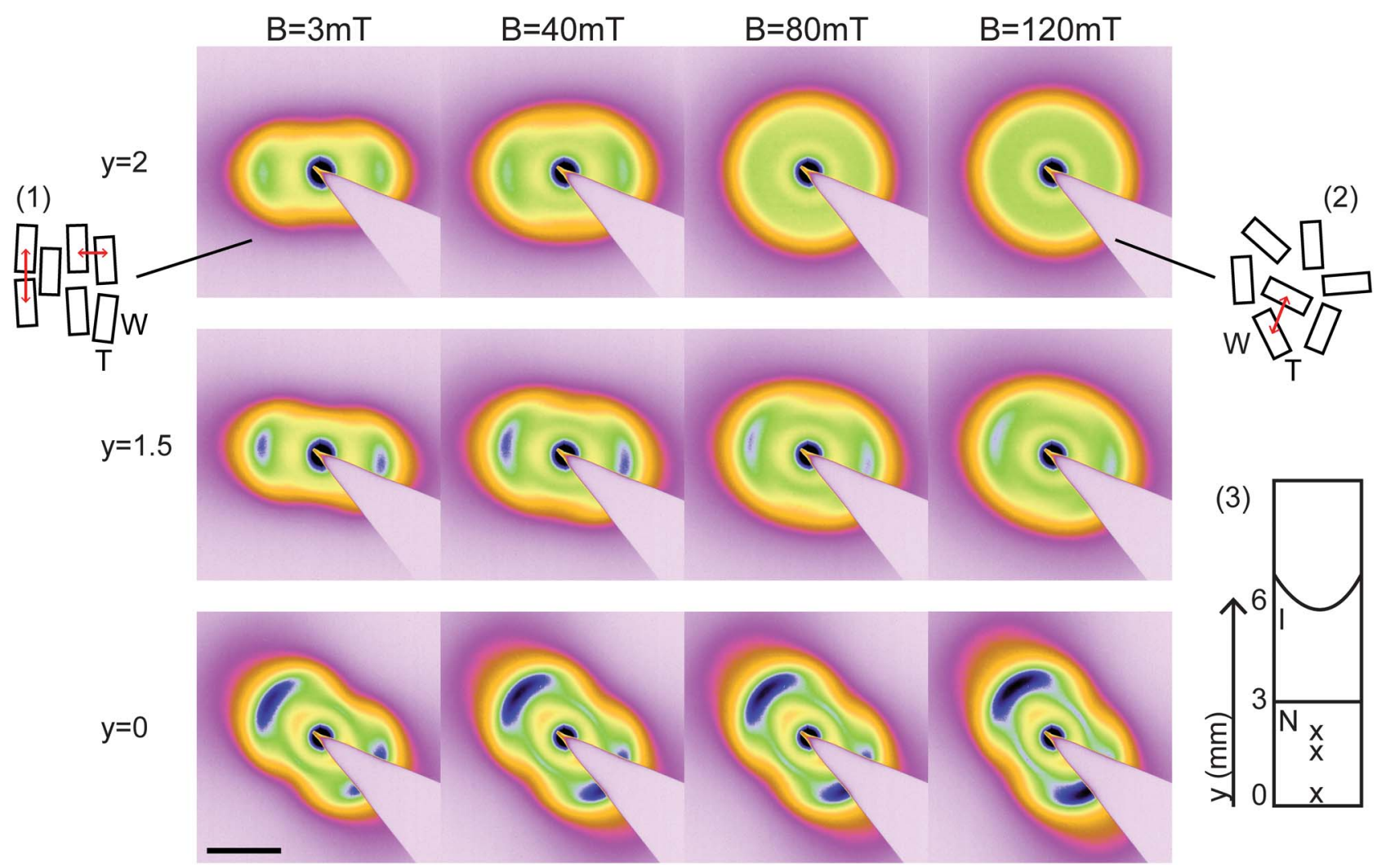

Fig. 2 SAXS patterns taken at three different heights in the capillary $(y=0,1.5$ and $2 \mathrm{~mm})$ submitted to an external magnetic field of $3,40,80$ and $120 \mathrm{mT}$, respectively. The magnetic field direction is parallel to the X-ray beam. (1 and 2) Schematic drawings illustrating the $N_{B}$ and $N_{+}$phase seen along the $L$-axis, respectively. (3) Schematic drawing of the capillary. Liquid crystal phases are denoted by I (isotropic) and $\mathrm{N}$ (nematic). The presented SAXS patterns were taken at the positions denoted by $x$. The black bar denotes $0.1 \mathrm{~nm}^{-1}$.

even almost identical. Additionally, the peaks of the independent $W$ and $T$ reflections are barely visible; they almost entirely fused to form one reflection at a $Q$-value in between $Q_{W}$ and $Q_{T}$. This is characteristic of the uniaxial nematic phase and confirms the $\mathrm{N}_{\mathrm{B}}$ to $\mathrm{N}_{+}$transformation. The azimuthal plots (Fig. 3(c)) show the loss of biaxial order with increasing field strength as well. Already at $40 \mathrm{mT}$ the peaks have become broader and at $120 \mathrm{mT}$ the different positional correlation peaks barely appear along orthogonal directions. For the profile taken at $Q_{W}$, the integrated intensity of the reflection is equal for the entire profile. The angular extension of the diffuse reflections indicates that the particles can now freely rotate around their $L$-axis, indicating $\mathrm{N}_{+}$ordering once more.

To confirm these observations the biaxiality was studied by applying the magnetic field in the direction perpendicular to the X-ray beam. SAXS patterns and the corresponding radial and azimuthal profiles are shown in Fig. 4 and 5 respectively. The patterns taken at $y=0 \mathrm{~mm}$ show a reflection at short $Q$ originating from the longest axis of the particles combined with a reflection at $Q=0.084 \mathrm{~nm}^{-1}$, originating from the particles' shortest dimension. Reflections of the $W$-correlation of the particles are not observed, thus confirming the biaxiality of the nematic phase. Upon increasing the magnetic field strength, the reflection of $T$ remains at fixed $Q$, confirming the biaxiality of the nematic phase at $y=0 \mathrm{~mm}$ in a magnetic field of $120 \mathrm{mT}$ (Fig. 5(b)).
The SAXS-pattern taken at $y=2 \mathrm{~mm}$ and $B=10 \mathrm{mT}$ shows the reflections of the $\mathrm{N}_{\mathrm{B}}$ phase: a weak reflection at short $Q$ originating from $L$ and in the orthogonal direction the $T$-reflection at $Q=0.083 \mathrm{~nm}^{-1}$. It is clear from the $I(Q)$ profiles of the patterns taken at $B=40$ and $120 \mathrm{mT}$ that the peak at large $Q$ is shifting to lower $Q$-values (Fig. 5(d)). This indicates that reflections are not solely originating from the $T$-dimension of the particles but from the $W$-dimension as well. This means that the particles are able to rotate around their long axis and are no longer biaxially ordered.

It is also interesting to investigate the influence of the magnetic field when the magnetic field strength is getting closer to the critical magnetic field strength $B^{*}$. It is known that, due to goethite's specific magnetic properties, particles in the nematic phase align their $L$-axis in weak magnetic fields $\left(B<B^{*}\right)$ but reorient to align their $T$-axis in strong magnetic fields $\left(B>B^{*}\right)$. As a result the $\mathrm{N}_{+}$phase transforms into a rotated $\mathrm{N}_{B}$ phase - where the particles' long axis is now aligned perpendicular to the field when submitted to a strong magnetic field. Here we observed the $\mathrm{N}_{\mathrm{B}}$ to $\mathrm{N}_{+}$transition in magnetic fields to be weak compared to the critical magnetic field, where the particles do not rotate yet and the induced magnetic moment does not have a significant influence on the phase behaviour. In Fig. 6 we present SAXS patterns with their corresponding azimuthal plots taken at $y=$ $2 \mathrm{~mm}$ in magnetic fields with field strengths $(B=160$ and $200 \mathrm{mT})$ closer to $B^{*}(250 \mathrm{mT})$. The profile of the pattern taken at 

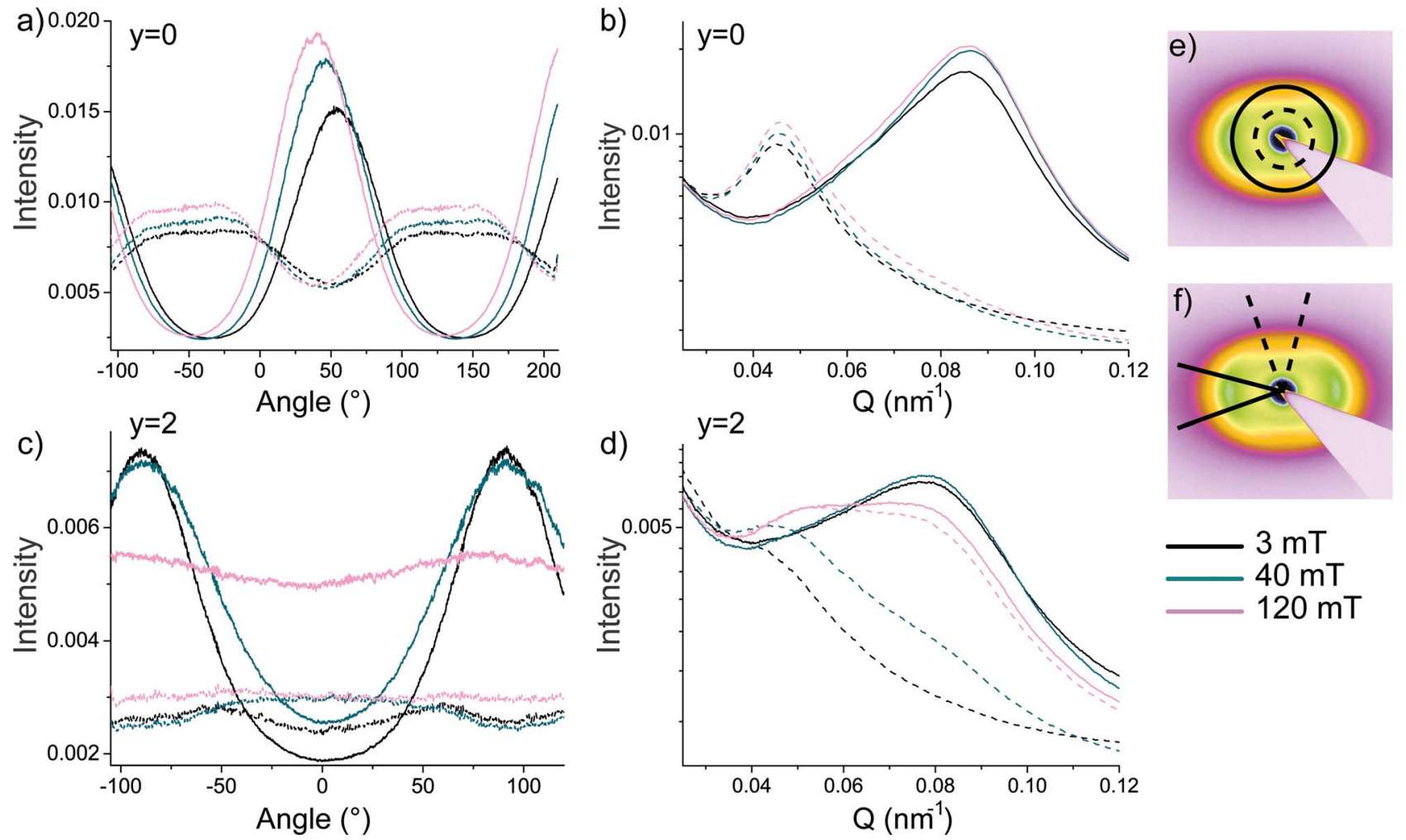
$120 \mathrm{mT}$

Fig. 3 Plots of selected SAXS patterns shown in Fig. 2 illustrating the decrease in biaxiality as a function of the height in the capillary $y$ and of the magnetic field strength. (a) and (c) show the azimuthal plots at $Q_{W}$ (dashed lines) and $Q_{T}$ (solid lines) for $y=0$ and $y=2 \mathrm{~mm}$ respectively (taken as illustrated in (e)). (b) and (d) show the radial profiles taken along two orthogonal directions corresponding to the $W$ (dashed lines) and $T$ (solid lines) reflections respectively (taken as illustrated in (f)).

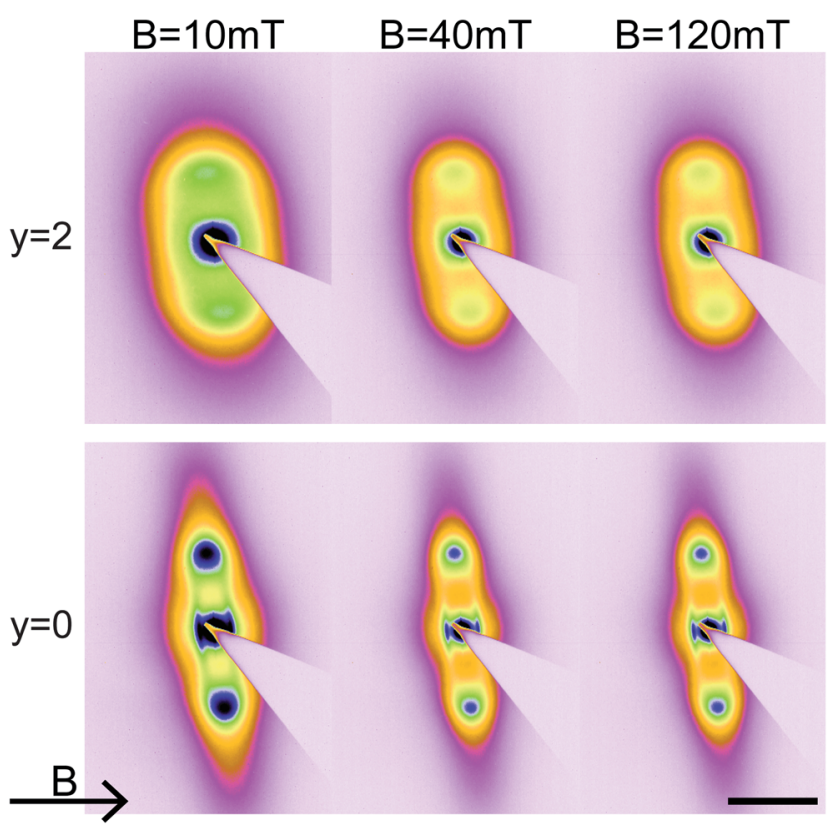

Fig. 4 SAXS patterns taken at two positions in the capillary $(y=0$ and $2 \mathrm{~mm}$ ), in a magnetic field varying from 10 to $120 \mathrm{mT}$. The magnetic field direction is perpendicular to the X-ray beam and is denoted by the black arrow. The black bar denotes $0.1 \mathrm{~nm}^{-1}$.
$B=160 \mathrm{mT}$ is similar to the profile at $120 \mathrm{mT}$, the differences are negligible. However if the profile corresponding to the pattern taken at $200 \mathrm{mT}$ is compared to the one of $160 \mathrm{mT}$, it appears that the amplitude of the peaks has increased. This suggests that the biaxial ordering slightly increased again. This might indicate that the perpendicular induced magnetic moment starts playing a role: the particles start aligning their $T$-axes as well thereby increasing the biaxial ordering of the board-like particles.

\section{Theoretical results}

The theoretical framework described in Section 3 was applied to predict phase diagrams for board-like particles submitted to an external magnetic field. Fig. 7 shows the well-known ${ }^{7,35}$ zerofield case as a function of the shape parameter $(v \equiv L / W-W / T)$ and goethite volume fraction $(\phi)$. A system of board-like particles undergoes a transition to a prolate uniaxial nematic phase $\left(\mathrm{N}_{+}\right)$if $v>0$, to an oblate uniaxial nematic $\left(\mathrm{N}_{-}\right)$if $v<0$ and to a biaxial nematic $\left(\mathrm{N}_{\mathrm{B}}\right)$ if $v=0$. Such isotropic-nematic phase transitions are in general discontinuous, albeit very weakly, and become continuous in a Landau critical point only if $v=0$. Here and in what follows we focus on low enough volume fractions so that the stability of inhomogeneous phases (e.g., smectic, crystals, ...) can be neglected.

The phase diagrams for board-like particles submitted to magnetic fields of intensity 3, 40, 80 and $120 \mathrm{mT}$ are shown in 

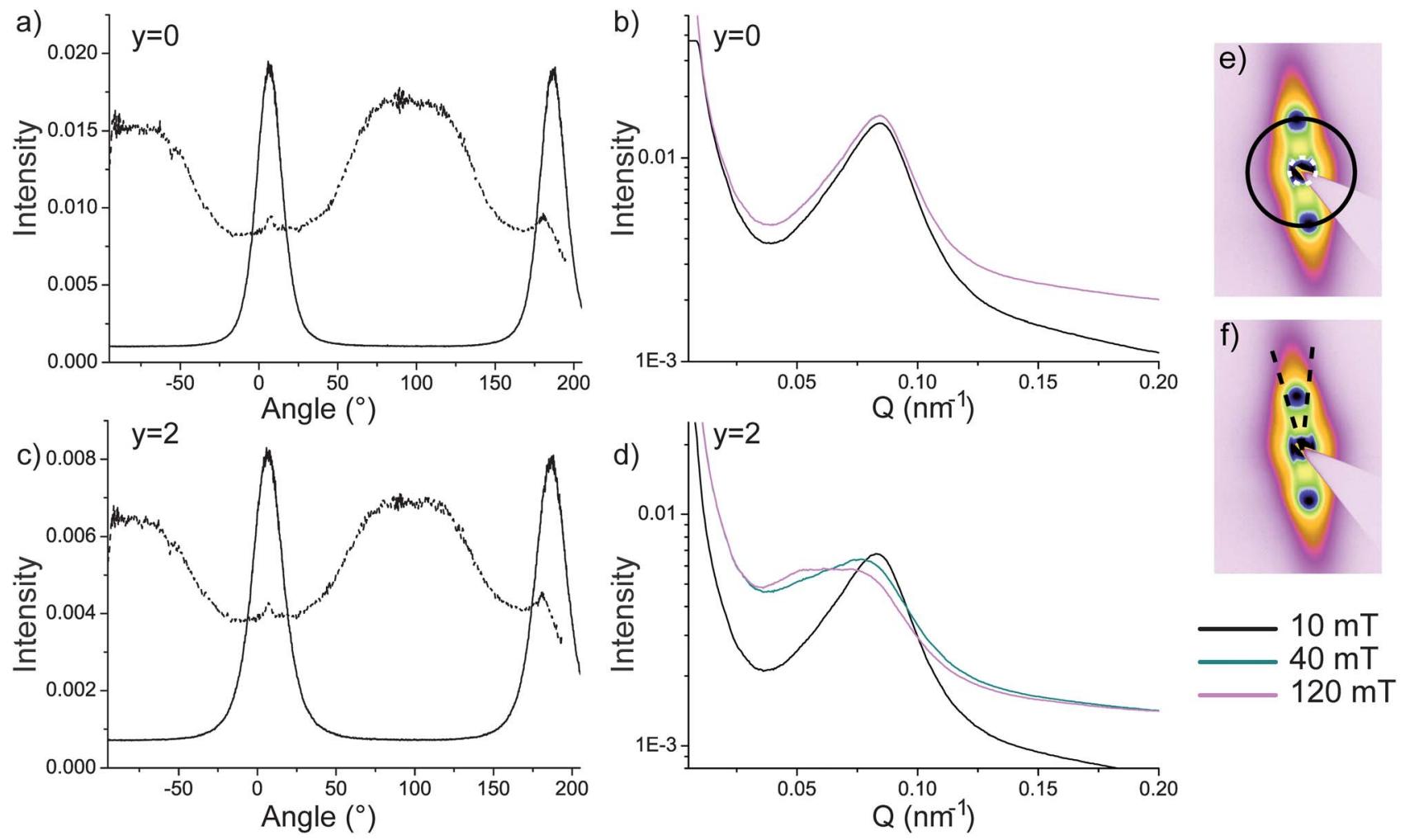

Fig. 5 Plots of selected SAXS patterns shown in Fig. 4 illustrating the decrease in biaxiality as a function of height in the capillary $y$ and the magnetic field strength. (a) and (c) show the azimuthal plots (taken as illustrated in (e)) at $Q_{T}\left(\sim 0.08 \mathrm{~nm}^{-1}\right.$ (solid lines)) and at $Q_{L}\left(\sim 0.017 \mathrm{~nm}^{-1}\right.$ (dashed lines)) for $y=0$ and $y=2 \mathrm{~mm}$ respectively in a $10 \mathrm{mT}$ magnetic field. Both plots show the biaxiality of the nematic phase. (b) and (d) show the radial profiles taken along the direction of the reflection at large $Q$ for 10, 40 and $120 \mathrm{mT}$ (taken as illustrated in (f)).

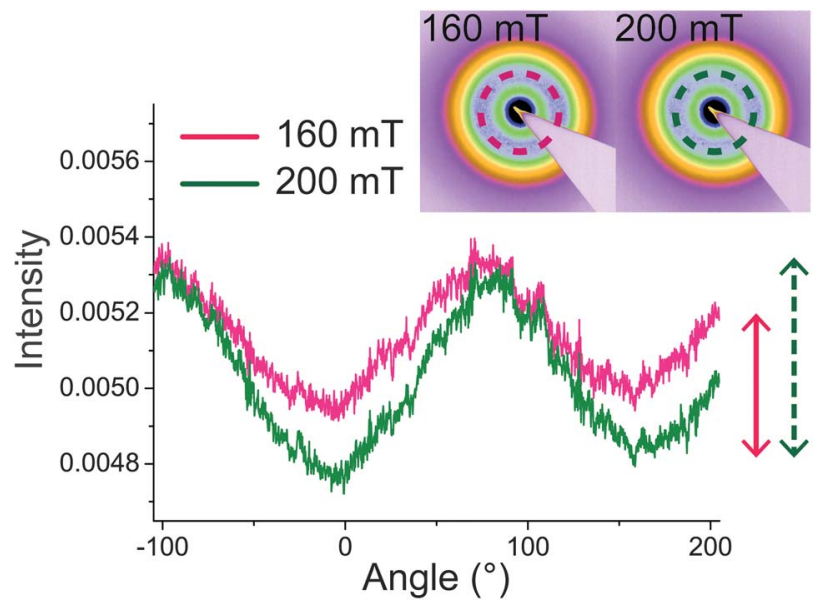

Fig. 6 Azimuthal plots of the SAXS patterns (inset) taken at $y=2$ in magnetic fields of 160 and $200 \mathrm{mT}$. The magnetic field direction is parallel to the X-ray beam. The integrated area is illustrated by the dashed circles in the inset. The solid and dashed arrows represent the peak height for the $160 \mathrm{mT}$ and the $200 \mathrm{mT}$ plots respectively. It is clear that the peak height of the $200 \mathrm{mT}$ plot is larger than the peak height of the $160 \mathrm{mT}$ plot. This indicates that biaxiality has increased again at $200 \mathrm{mT}$.

Fig. 8. For comparison the non-zero field case is shown by the white lines. Let us first focus on Fig. 8(a), which reports the phase diagram of board-like particles in the presence of a 3

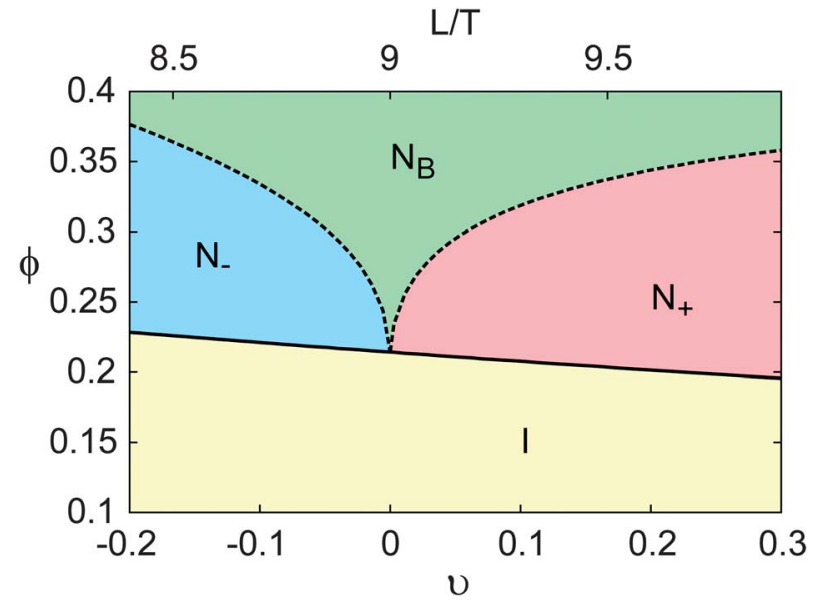

Fig. 7 Phase diagram of magnetic board-like particles in the absence of a magnetic field. $\phi=$ volume fraction. Here and in the following plots the shape parameter $v(\equiv L / W-W / T)$ is modified by changing the aspect ratio $L / T$ while keeping $W / T$ fixed at 3 . Solid lines indicate (very weakly) first order transitions, whereas dashed lines indicate continuous transitions. Liquid crystal phases are denoted by I (isotropic), $\mathrm{N}_{-}$, $\mathrm{N}_{+}$and $\mathrm{N}_{\mathrm{B}}$ (oblate uniaxial, prolate uniaxial and biaxial nematic, respectively).

mT magnetic field. In spite of the low field strength, the effect of the explicit breaking of the rotational symmetry due to the field is already evident. First, due to the coupling between the 
permanent magnetic dipole and the field, the particles acquire a weak tendency to align their long axis $L$ along the field even at very low volume fraction. As a consequence, the symmetry of the low-density isotropic phase (Fig. 7) becomes the same as that of the prolate uniaxial nematic phase (red region in Fig. 7 and 8). Such a phenomenon closely resembles the phase behaviour of rods under the effect of an external magnetic field which couples to the orientation of their main axis. ${ }^{36}$ In the latter context, the low-density phase which acquires the same symmetries as the higher-density nematic phase is termed "para-nematic". The distinction between a nematic and a para-nematic phase is needed as long as the two phases are separated by a discontinuous phase transition. This happens to be the case as long as the field strength is sufficiently low. However, at high enough field strength such a phase transition becomes continuous, and the two phases merge into the same nematic phase. ${ }^{36}$ As pointed out in the analysis of the zero-field case ( $c f$. Fig. 7), in the present model the isotropic-nematic phase transition of board-like particles is very weakly discontinuous. This means that at magnetic field strength as low as $3 \mathrm{mT}$ this phase transition is already continuous, and the distinction between para-nematic and nematic phases is pointless. The second manifest effect of the external field on the phase behaviour of board-like particles is the disappearance of the oblate uniaxial nematic phase (blue region in Fig. 7) in favour of the biaxial nematic (green region in Fig. 7 and 8). It is important to realize that at such a low magnetic field the deviations from the zero-field case play the role of a very weak symmetry-breaking perturbation. This statement is confirmed by the fact that the transition line (black dashed line) between the only two phases left in the phase diagram, that is the $\mathrm{N}_{+}$and the $\mathrm{N}_{\mathrm{B}}$, does not change appreciably from the zero-field case. In contrast, relevant deviations in the $\mathrm{N}_{+}-$ $\mathrm{N}_{\mathrm{B}}$ transition develop at higher magnetic field strengths. The phase diagram in Fig. 8(b) shows that already at $40 \mathrm{mT}$ the original $\mathrm{N}_{\mathrm{B}}$ phase formed close to the Landau critical point, where $\phi$ is relatively low, has transformed into a $\mathrm{N}_{+}$phase. The phase diagrams for magnetic fields of 80 and $120 \mathrm{mT}$ (Fig. 8(c) and (d)) show that the $\mathrm{N}_{\mathrm{B}}-\mathrm{N}_{+}$transition line shifts to higher volume fractions with increasing magnetic field strengths. This indicates that more and more of the original $\mathrm{N}_{\mathrm{B}}$ phase is transformed into a $\mathrm{N}_{+}$phase.
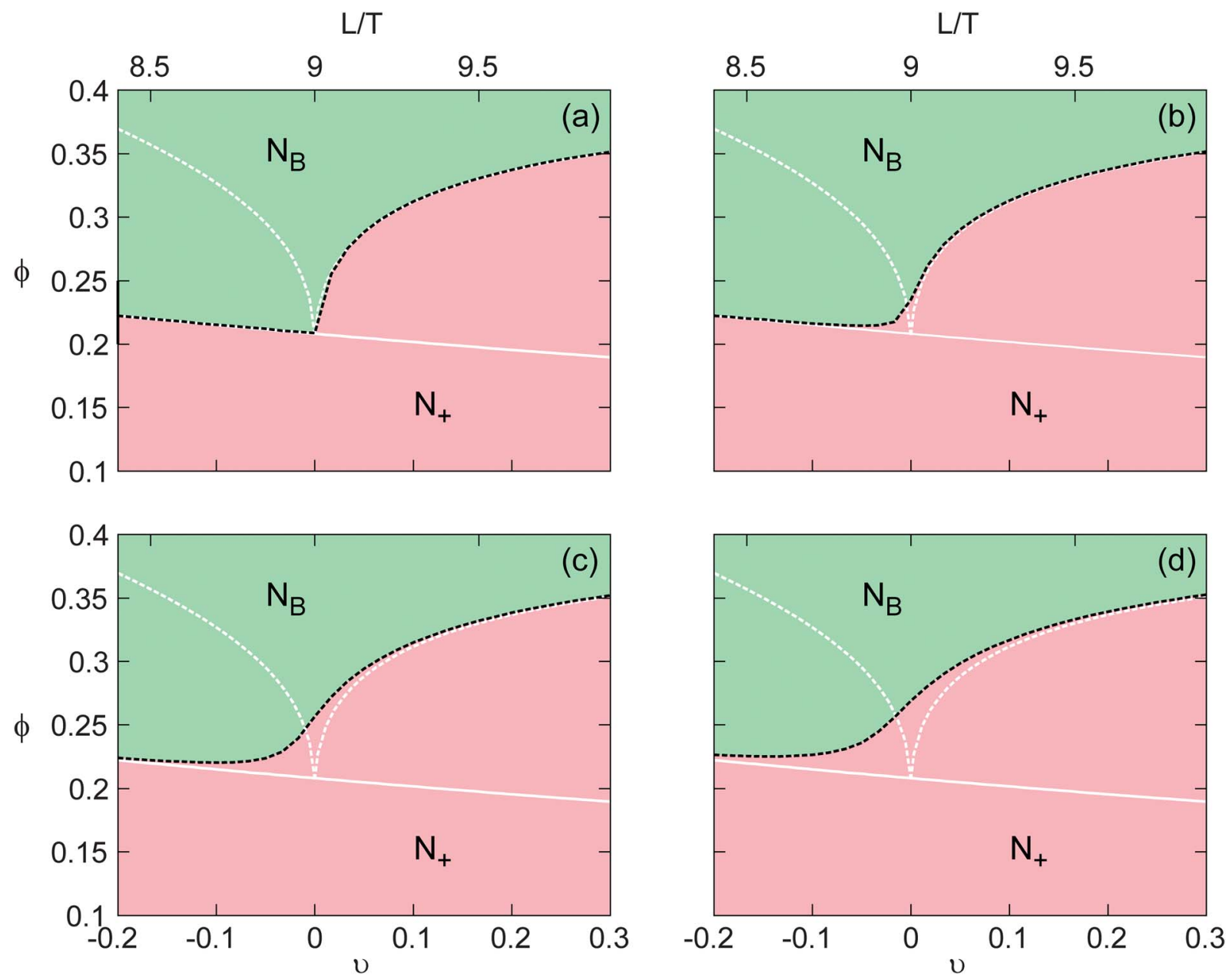

Fig. 8 Theoretical prediction of phase diagrams of magnetic board-like particles in a magnetic field of (a) $3 \mathrm{mT}$, (b) $40 \mathrm{mT}$, (c) $80 \mathrm{mT}$ and (d) 120 $\mathrm{mT}$ under the effect of the external potential in eqn (8). White lines represent the zero-field phase diagram. $N_{+}$and $N_{B}$ denote the prolate uniaxial and biaxial nematic liquid crystal phase, respectively. 


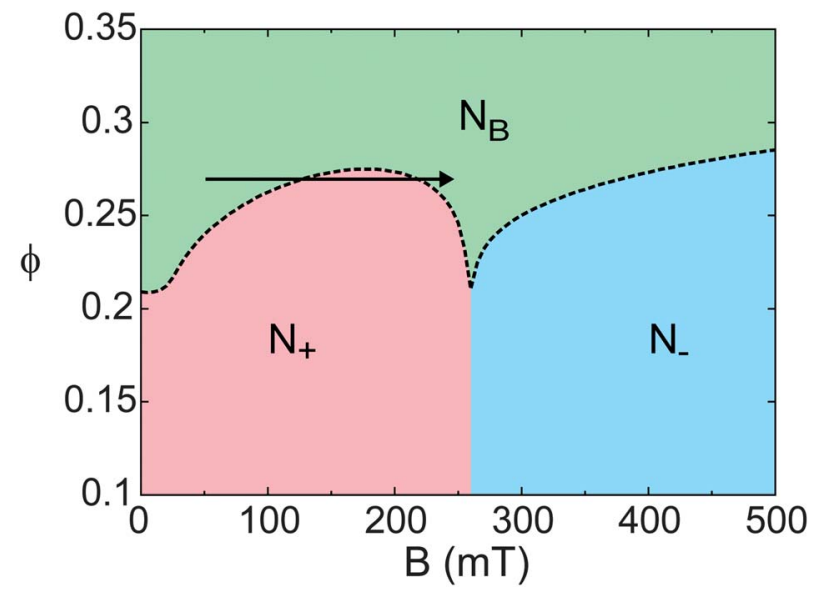

Fig. 9 Theoretical prediction of the phase diagram of magnetic board-like particles with shape parameter $v=0(L \times W \times T=9 \times 3 \times$ 1) as a function of volume fraction $\phi$ and magnetic field strength. The black arrow illustrates the re-entrant $N_{B}$ to $N_{+}$to $N_{B}$ transition with increasing field strength.

An alternative way to visualize the data is offered in Fig. 9, where we report the phase diagram of a system of board-like particles with fixed shape parameter $v=0(L / T=9 ; W / T=3)$ as a function of the magnetic field strength. This plot shows explicitly the variation of the $\mathrm{N}_{\mathrm{B}}$ to $\mathrm{N}_{+}$transition with the intensity of the field. The transition line shows that the magnetic field strength needed to induce the $\mathrm{N}_{+}$phase increases with increasing volume fraction. Above a certain volume fraction the particles remain in the $\mathrm{N}_{\mathrm{B}}$ phase for all field strengths lower than $B^{*}$. These theoretical predictions agree with the experimental observations of the field-induced $\mathrm{N}_{\mathrm{B}}$ to $\mathrm{N}_{+}$ transition. Our experimental results also indicated that the biaxial order is restored when the magnetic field strength approaches the critical magnetic field strength $\left(B^{*}=250 \mathrm{mT}\right)$. Also this is supported by theory. The black arrow in Fig. 9 clearly shows the re-entrant $\mathrm{N}_{\mathrm{B}}$ to $\mathrm{N}_{+}$to $\mathrm{N}_{\mathrm{B}}$ transition. Interestingly, Fig. 9 also shows that for field strengths above $B^{*}$, where alignment along the short axis $T$ is favoured, the $\mathrm{N}_{\mathrm{B}}$ phase again loses biaxial order but now in favour of oblate uniaxial ordering.

It is crucial to keep in mind that in the present model the $\mathrm{N}_{\mathrm{B}^{-}}$ $\mathrm{N}_{+}$transition is continuous. This means that the biaxial ordering and the prolate uniaxial ordering close to the transition line are weak, hence those phases do not differ as drastically from each other as appears from the phase diagram at first glance. This is also emphasized by the order parameter plots shown in Fig. 10. At a volume fraction of $\sim 0.23$ the $\mathrm{N}_{\mathrm{B}}$ phase transforms into the $\mathrm{N}_{+}$phase in a field of $40 \mathrm{mT}$ for particles with $v=0$ (Fig. 8(b) and 9). It is however apparent from the order parameter plots (Fig. 10(b)) that the corresponding decrease in the biaxial nematic order parameter is not drastic upon going from 3 to $40 \mathrm{mT}$. The biaxial order parameter decreases with increasing $B$ and at high enough field strengths, e.g. $80 \mathrm{mT}$ for a volume fraction of 0.23 , eventually becomes zero, indicating that the particles are no longer biaxially ordered. The uniaxial order parameter along $L$ plotted in Fig. 10(a), in contrast to the biaxial order parameter, increases
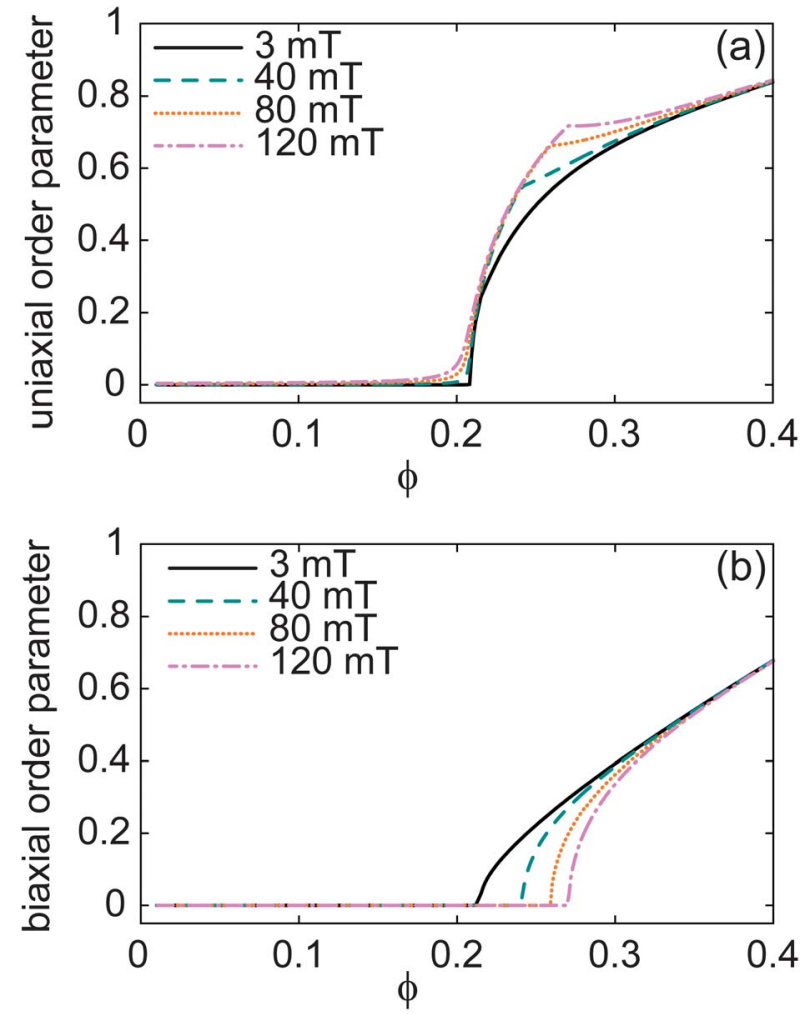

Fig. 10 (a) Uniaxial order parameter and (b) biaxial order parameter plotted as a function of volume fraction $\phi$ for four magnetic field strengths $(3,40,80$ and $120 \mathrm{mT}$ respectively) for particles with $v=$ $0(L \times W \times T=9 \times 3 \times 1)$.

with increasing field strength. The discontinuities in the curves' first derivatives represent the $\mathrm{N}_{+}-\mathrm{N}_{\mathrm{B}}$ transition points.

The theoretical predictions of the influence of the magnetic field on the other liquid crystal phases are also interesting. As expected, the phase diagrams in Fig. 8 show that the magnetic field breaks the symmetry of the isotropic phase and induces the $\mathrm{N}_{+}$phase, which in turn transforms into the $\mathrm{N}_{-}$phase for $B>$ $B^{*}$ (Fig. 9). Especially interesting is the influence of the magnetic field on the $\mathrm{N}_{-}$phase, which is expected at zero field for particles with $v<0$. The phase diagrams in Fig. 8 clearly show that the $\mathrm{N}_{-}$phase transforms into the rare biaxial nematic phase under the influence of a magnetic field. Alignment of the particles' $L$-axes in the field breaks the symmetry of the $\mathrm{N}_{-}$ phase which leads to the formation of the $\mathrm{N}_{B}$ phase. Again, one has to realize that the induced $\mathrm{N}_{\mathrm{B}}$ phase in a $3 \mathrm{mT}$ magnetic field has very weak biaxial ordering; the corresponding biaxial order parameter (not shown) increases with increasing field strength.

\section{Conclusions and outlook}

Using SAXS we observed that goethite particles in the $\mathrm{N}_{\mathrm{B}}$ phase lose their biaxial ordering upon applying a magnetic field along the main director of the $\mathrm{N}_{B}$ phase. The extent of the loss of biaxial ordering strongly depends on the strength of the magnetic field. The field-induced $\mathrm{N}_{\mathrm{B}}$ to $\mathrm{N}_{+}$transition can be 
understood in terms of the entropy balance that drives lyotropic liquid crystal formation. The stability of the $\mathrm{N}_{B}$ phase can be interpreted as a balanced competition between rod-like $\left(\mathrm{N}_{+}\right)$and plate-like $\left(\mathrm{N}_{-}\right)$behaviour. The interaction of the applied magnetic field with the permanent magnetic moment along the $L$-axis of the particles substantially narrows the orientation distribution function which describes the orientation of the particles. 'Fixing' the $L$-axis of the particles in the field releases more space for the particles to rotate freely along their $L$-axis. This increases the orientational entropy and pushes the particles towards $\mathrm{N}_{+}$ordering.

We additionally found that the extent of the loss of biaxial ordering strongly depends on the goethite volume fraction $\phi$. At high volume fractions the field-induced observed decrease in biaxiality is significantly less, and is even negligible for sufficiently high $\phi$. This can be explained by the excluded-volume entropy that favours $\mathrm{N}_{\mathrm{B}}$ ordering and whose contribution to the total entropy strongly increases with increasing volume fraction.

The loss of biaxial ordering persists up to a field strength of $B \approx 160 \mathrm{mT}$. For higher magnetic field strengths, closer to $B^{*}$, the biaxial order was found to increase again as a result of alignment of the particles' $T$-axes due to the increasing induced magnetic moment.

Theoretically the influence of a magnetic field was studied using a mean-field theory in which the magnetic properties of the particles were modelled by taking into account the effect of both the permanent and the induced magnetic dipoles. The theoretical results agree well with the experimental findings in spite of the approximations required. Moreover, polydispersity, which is known to have a significant influence on goethite phase behaviour, ${ }^{12,16,25,37}$ is not accounted for in the present analysis. Therefore the absolute values given in the theoretical phase diagrams and the order parameter plots presented in this paper are not expected to be quantitatively trustworthy. Nevertheless, the theory gives reliable qualitative predictions on the effect of the magnetic field on the phase behaviour of magnetic board-like particles.

Interestingly, the theory also predicts that the rare $\mathrm{N}_{\mathrm{B}}$ phase can be formed upon applying a magnetic field to a $\mathrm{N}_{-}$ordered phase of particles with a negative shape parameter. This magnetic-field induced $\mathrm{N}_{B}$ phase opens up new possibilities. Normally a very specific shape of (goethite) board-like particles is required $(L / W=W / T)$ to create a biaxial nematic phase. There is a small $\mathrm{N}_{\mathrm{B}}$ window around $v=0$ that will be truncated by the smectic phase at higher volume fractions. ${ }^{38}$ However, the shape of the particles is an experimental parameter that is hard to tune..$^{39}$ Our new findings predict that the rare $\mathrm{N}_{\mathrm{B}}$ phase can easily be induced by applying a magnetic field with a carefully chosen field strength to a $\mathrm{N}_{-}$phase. The magnetic field strength is a parameter that is easy to tune and the field-induced $\mathrm{N}_{-}$to $\mathrm{N}_{\mathrm{B}}$ transition is not limited to the goethite system presented here. Our theoretical work implies that for any system of boardlike particles that carry a magnetic (or electric) dipole along their longest axis and favours $\mathrm{N}_{-}$ordering in zero field, the $\mathrm{N}_{\mathrm{B}}$ phase can be induced by applying a weak magnetic (or electric) field.
It is also worth mentioning that our theoretical model indicates that it is not necessarily true that the $T$-axis aligns in a strong magnetic field because it is the magnetic easy axis, which has generally been assumed. ${ }^{18,20-23}$ This assumption was always made from the experimental observation that goethite particles in the nematic phase align along $T$ in a strong magnetic field. However, in the nematic phase other factors like the excluded volume entropy have a strong influence on the alignment of the particles. The theoretical work presented in this paper shows that even when the magnetic susceptibilities along $W$ and $T$ are equal, particles align along $T$ since alignment along $W$ is strongly suppressed due to the excluded volume interactions. For future work it is therefore interesting, although challenging, to investigate the alignment of particles in very dilute suspensions to exclude the effects of multiple-particle interactions.

To summarize, the results presented in this paper show that a magnetic field is a useful 'tool' to control nematic phases of board-like particles. Carefully choosing the volume fraction and the magnetic field strength, parameters that are both easy to tune, enables the formation of the $\mathrm{N}_{\mathrm{B}}$ phase (out of $\mathrm{N}_{-}$) as well as the $\mathrm{N}_{+}$phase (out of $\mathrm{N}_{\mathrm{B}}$ ). Additionally, Belli et al. ${ }^{17}$ already theoretically predicted that the addition of a depletant can induce both the $\mathrm{N}_{\mathrm{B}}$ and $\mathrm{N}_{-}$phase in systems of board-like particles with a positive shape parameter. Both options open up possibilities to engineer the desired character of the nematic phase for a range of particle shapes. This is especially useful since the rare biaxial nematic phase can now be induced.

\section{Acknowledgements}

This work is part of the research programme SFB TR6 and the FOM-projectruimte of the 'Stichting voor Fundamenteel Onderzoek der Materie (FOM)', which is financially supported by the 'Nederlandse Organisatie voor Wetenschappelijk Onderzoek (NWO)' and the 'Deutsche Forschungsgemeinschaft (DFG)'. We would like to thank the staff of the BM26 beamline "DUBBLE" at the ESRF, as well as Vera Meester and Antara Pal for their support during the synchrotron experiments.

\section{Notes and references}

1 D. K. Yang and S. T. Wu, in Fundamentals of Liquid Crystal Devices, Wiley, 2006, p. 378.

2 J. Gabriel and P. Davidson, Adv. Mater., 2000, 12, 9.

3 H. N. W. Lekkerkerker and G. J. Vroege, Philos. Trans. R. Soc., A, 2013, 371, 20120263.

4 L. Onsager, Ann. N. Y. Acad. Sci., 1949, 51, 627-659.

5 M. Freiser, Phys. Rev. Lett., 1970, 24, 1041.

6 R. Alben, Phys. Rev. Lett., 1973, 30, 778-781.

7 J. Straley, Phys. Rev. A: At., Mol., Opt. Phys., 1974, 10, 18811887.

8 L. Yu and A. Saupe, Phys. Rev. Lett., 1980, 45, 1000-1003.

9 J. Lee, T. Lim, W. Kim and J. Jin, J. Appl. Phys., 2007, 101, 034105.

10 Y. Galerne, A. Neto and L. Liebert, J. Chem. Phys., 1987, 87, 1851-1855. 
11 E. van den Pol, A. V. Petukhov, D. M. E. Thies-Weesie, D. V. Byelov and G. J. Vroege, Phys. Rev. Lett., 2009, 103, 258301.

12 E. van den Pol, A. A. Verhoeff, A. Lupascu, M. A. Diaconeasa, P. Davidson, I. Dozov, B. W. M. Kuipers, D. M. E. ThiesWeesie and G. J. Vroege, J. Phys.: Condens. Matter, 2011, 23, 194108.

13 E. van den Pol, D. M. E. Thies-Weesie, A. V. Petukhov, D. V. Byelov and G. J. Vroege, Liq. Cryst., 2010, 37, 641-651.

14 J. P. F. Lagerwall and G. Scalia, Curr. Appl. Phys., 2012, 12, 1387-1412.

15 P. Camp and M. Allen, J. Chem. Phys., 1997, 106, 6681-6688.

16 S. Belli, A. Patti, M. Dijkstra and R. van Roij, Phys. Rev. Lett., 2011, 107, 148303.

17 S. Belli, M. Dijkstra and R. van Roij, J. Phys.: Condens. Matter, 2012, 24, 284128.

18 B. Lemaire, P. Davidson, J. Ferre, J. Jamet, P. Panine, I. Dozov and J. Jolivet, Phys. Rev. Lett., 2002, 88, 125507.

19 B. Lemaire, P. Davidson, J. Ferre, J. Jamet, D. Petermann, P. Panine, I. Dozov and J. Jolivet, Eur. Phys. J. E, 2004, 13, 291-308.

20 B. Lemaire, P. Davidson, J. Ferre, J. Jamet, D. Petermann, P. Panine, I. Dozov, D. Stoenescu and J. Jolivet, Faraday Discuss., 2005, 128, 271-283.

21 B. Lemaire, P. Davidson, D. Petermann, P. Panine, I. Dozov, D. Stoenescu and J. Jolivet, Eur. Phys. J. E, 2004, 13, 309-319.

22 E. van den Pol, A. Lupascu, M. A. Diaconeasa, A. V. Petukhov, D. V. Byelov and G. J. Vroege, J. Phys. Chem. Lett., 2010, 1, 2174-2178.

23 E. van den Pol, A. V. Petukhov, D. V. Byelov, D. M. E. ThiesWeesie, A. Snigirev, I. Snigireva and G. J. Vroege, Soft Matter, 2010, 6, 4895-4899.
24 E. van den Pol, A. Lupascu, P. Davidson and G. J. Vroege, J. Chem. Phys., 2010, 133, 164504.

25 E. van den Pol, D. M. E. Thies-Weesie, A. V. Petukhov, G. J. Vroege and K. Kvashnina, J. Chem. Phys., 2008, 129, 164715. 26 A. Petukhov, J. Thijssen, D. t. Hart, A. Imhof, A. van Blaaderen, I. Dolbnya, A. Snigirev, A. Moussaid and I. Snigireva, J. Appl. Crystallogr., 2006, 39, 137-144.

27 W. Bras, I. Dolbnya, D. Detollenaere, R. van Tol, M. Malfois, G. Greaves, A. Ryan and E. Heeley, J. Appl. Crystallogr., 2003, 36, 791-794.

28 R. Zwanzig, J. Chem. Phys., 1963, 39, 1714.

29 K. Shundyak and R. van Roij, Phys. Rev. B: Condens. Matter Mater. Phys., 2004, 69, 041703.

30 R. Evans, Adv. Phys., 1979, 28, 143-200.

31 S. Lee, J. Chem. Phys., 1988, 89, 7036-7037.

32 S. Lee, J. Chem. Phys., 1987, 87, 4972-4974.

33 J. Parsons, Phys. Rev. A: At., Mol., Opt. Phys., 1979, 19, 12251230.

34 E. van den Pol, Goethite liquid crystals and magnetic field effects, Utrecht University, 2010.

35 B. Mulder, Phys. Rev. A: At., Mol., Opt. Phys., 1989, 39, 360370.

36 A. Khoklov and A. Semenov, Macromolecules, 1982, 15, 12721277.

37 A. B. G. M. Leferink op Reinink, E. van den Pol, D. V. Byelov, A. V. Petukhov and G. J. Vroege, J. Phys.: Condens. Matter, 2012, 24, 464127.

38 M. Taylor and J. Herzfeld, Phys. Rev. A: At., Mol., Opt. Phys., 1991, 44, 3742-3751.

39 D. M. E. Thies-Weesie, J. P. de Hoog, M. H. H. Mendiola, A. V. Petukhov and G. J. Vroege, Chem. Mater., 2007, 19, 5538-5546. 